Groups Geom. Dyn. 8 (2014), 331-353

DOI $10.4171 / \mathrm{GGD} / 228$
Groups, Geometry, and Dynamics

(C) European Mathematical Society

\title{
A horospherical ratio ergodic theorem for actions of free groups
}

\author{
Lewis Bowen and Amos Nevo*
}

\begin{abstract}
We prove a ratio ergodic theorem for discrete non-singular measurable equivalence relations, provided they satisfy a strong form of the Besicovich covering property. In particular, this includes all hyperfinite measurable equivalence relation. We then use this result to study general non-singular actions of non-abelian free groups and establish a ratio ergodic theorem for averages along horospheres.
\end{abstract}

Mathematics Subject Classification (2010). 37A15, 37A30.

Keywords. Ratio ergodic theorem, free groups.

\section{Introduction}

Consider a non-singular action of a countable group $G$ on a standard $\sigma$-finite measure space $(X, \mathcal{B}, \eta)$, which we denote by $x \mapsto T^{g} x$. From the action on $X$, there is an induced isometric action on $L^{\infty}(X, \eta)$ also denoted by $T^{g}$, given by $T^{g} f(x)=$ $f\left(T^{g^{-1}} x\right)$. This induces an isometric action on the Banach pre-dual of $L^{\infty}(X, \eta)$, namely on $L^{1}(X, \eta)$ which is given by $\widehat{T}^{g}(f)=T^{g} f \cdot \frac{d g \eta}{d \eta}$.

This set-up gives rise to a wide array of important and interesting examples of actions, where one would like to study the statistical properties of the distribution of the orbits of $G$ in $X$. Very little is known about this problem for general groups, and let us begin by reviewing the main results in the case of actions of abelian groups.

1.1. The ratio ergodic theorem for commuting transformations. For $\mathbb{Z}$-actions there is a generalization of Birkhoff's pointwise ergodic theorem, in the form of a ratio ergodic theorem. This generalization was initiated by Hopf [Ho37], and was later generalized further by Hurewicz [Hu44] and extended to operators by ChaconOrnstein [CO60]. The ratio ergodic theorem states that if $T:(X, \lambda) \rightarrow(X, \lambda)$ is a non-singular and conservative transformation, $u, v \in L^{1}(X, \lambda)$ and $v>0$ then the

*First-named author supported in part by NSF grant DMS-1000104 and BSF grant 2008274. Secondnamed author supported in part by ISF, and BSF grant 2008274 
ratios

$$
\operatorname{RATIO}_{n}[u, v]:=\frac{\sum_{k=0}^{n} \widehat{T}^{k} u}{\sum_{k=0}^{n} \widehat{T}^{k} v}
$$

converge pointwise almost everywhere as $n \rightarrow \infty$ to a function $r(u, v)$ on $X$ satisfying

- $r(u, v) \circ T=r(u, v)$, namely $r(u, v)$ is $T$-invariant,

- $\int f \cdot r(u, v) v d \lambda=\int f \cdot u d \lambda$ for any $f \in L^{\infty}(X, \lambda)$ such that $f \circ T=f$.

In particular, if $T$ is ergodic then $r(u, v)$ equals the constant $\frac{\int u d \lambda}{\int v d \lambda}$ almost everywhere. In general,

$$
r(u, v)=\mathbb{E}_{\lambda_{v}}\left[\frac{u}{v} \mid \mathcal{I}\right]
$$

is the conditional expectation of $\frac{u}{v}$ on the $\sigma$-algebra $\mathcal{I}$ of $T$-invariant sets with respect to the measure $\lambda_{v}$ defined by $d \lambda_{v}=v d \lambda$.

This result has only recently been extended to $\mathbb{Z}^{d}$ actions by Feldman [Fe07] and by Hochman [Ho10]. As to non-amenable groups, let us first recall some of the original motivating problems, and then describe in the next section some recent examples of ratio ergodic theorems and also some counterexamples.

1.2. Ratio equidistribution for non-commuting transformations. A significant source of important problems arises already in the special case in which $(X, \mathcal{B}, \lambda)$ is a locally compact group with Haar measure, $\phi: \Gamma \rightarrow X$ is a group homomorphism onto a dense subgroup, the action $\left(T^{g}\right)_{g \in \Gamma}$ is given by $T^{g}(x)=\phi(g) x$ and $u, v$ are compactly supported continuous functions. In this case, it is natural to ask whether the ratios $\mathrm{RATIO}_{n}[u, v]$ converge everywhere (instead of almost everywhere).

Arnol'd and Krylov were among the first to consider the problem of establishing equidistribution for actions of free groups [AK63]. They established uniform convergence in the case when the group $X$ is $\mathrm{SO}_{3}(\mathbb{R})$, but the same method applies whenever the group $X$ is compact and connected. We note that in the case that the underlying invariant measure is finite the mean and subsequently the pointwise ergodic theorem for completely general actions of free groups were considered by several authors [Gu69], [Gr99], [Ne94], [NS94], [Bu02], [BN13a] (see also the recent survey [BK12]).

Kazhdan considered equidistribution of ratios in the case when $X$ is the isometry group of the Euclidean plane and the averages are random walk averages [Ka65]. His argument was corrected and the results extended by Guivarc'h [Gu76] (see also [Vo04]). An important advance in the case when $X$ equals the isometry group of Euclidean $n$-space was very recently obtained by Varju [Va12]. Breuillard has obtained positive results when $X$ is the Heisenberg group [Br05] and the averages are random walk averages. He has also obtained positive results when $X$ is any simply-connected nilpotent Lie group [Br10] and the averages are uniform over balls in the Cayley graph of the image group $\phi(\Gamma)$. A survey of these results can be found in [Br06]. 


\section{Some recent developments, and statement of the main result}

2.1. Some recent ratio ergodic theorems. Let us now describe some developments over the last decade, which will illuminate the subtlety of the problem of establishing ratio ergodic theorems and some of the difficulties that must be overcome in the nonamenable context. We will describe results pertaining to actions of a lattice subgroup $\Gamma$ of a semisimple algebraic group $G$ on homogeneous spaces of $G$. In order to keep the exposition focused, let us concentrate on the case $G=\mathrm{SL}_{2}(\mathbb{R})$, although all the results we will describe apply in much greater generality. Let $\Gamma \subset \mathrm{SL}_{2}(\mathbb{R})$ be any lattice subgroup, for example a lattice isomorphic to the free group $\mathbb{F}_{r}$.

2.1.1. Lattice action on the boundary of the hyperbolic plane. Consider the group $\mathrm{SL}_{2}(\mathbb{R})$ and its maximal compact subgroup $K=\mathrm{SO}_{2}(\mathbb{R})$. The homogeneous space $G / K$ can be identified with the hyperbolic plane $\mathbb{H}^{2}$. Fix the point $o=[K] \in$ $G / K=\mathbb{H}^{2}$, and the $G$-invariant Riemannian metric $d$ on $\mathbb{H}^{2}$.

The lattice $\Gamma$ acts on the homogeneous space $B=P \backslash \mathrm{SL}_{2}(\mathbb{R})=P^{1}(\mathbb{R})$ where $P$ is the group of lower triangular matrices, and denote by $m$ the unique $K$-invariant probability measure on $B$. For any line $[v] \in B$, and any two continuous functions $\phi$ and $\psi$ on $B$, with $\int_{B} \psi d m \neq 0$ the following holds:

$$
\lim _{T \rightarrow \infty} \frac{\sum_{d(\gamma o, o) \leq T} \phi([v] \gamma)}{\sum_{d(\gamma o, o) \leq T} \psi([v] \gamma)}=\frac{\int_{B} \phi([w]) d m([w])}{\int_{B} \psi([w]) d m([w])} .
$$

This result is based on a precise asymptotics established in [G03] for the expression $\sum_{d(\gamma o, o) \leq T} \phi([v] \gamma)$, from which the ratio equidistribution theorem is an immediate corollary. The lattice action on the boundary $B=P \backslash G$ was originally considered (in greater generality) by Gorodnik [G03], and developed further in GorodnikMaucourant [GM05] and Gorodnik-Oh [GO07].

2.1.2. Lattice action on the Euclidean plane. Consider the homogeneous space $\mathbb{R}^{2} \backslash\{0\}=N \backslash \mathrm{SL}_{2}(\mathbb{R}), N$ the lower triangular unipotent group. Fix any norm on $M_{2}(\mathbb{R})$. Let $v \in \mathbb{R}^{2}$ be any vector whose $\Gamma$-orbit is dense in $\mathbb{R}^{2}$. Then given any two continuous compactly supported functions $\phi, \psi$ on $\mathbb{R}^{2}$,

$$
\lim _{T \rightarrow \infty} \frac{\sum_{\|\gamma\| \leq T} \phi(v \gamma)}{\sum_{\|\gamma\| \leq T} \psi(v \gamma)}=\frac{\int_{\mathbb{R}^{2}} \phi(w) \alpha_{v}(w) d w}{\int_{\mathbb{R}^{2}} \psi(w) \alpha_{v}(w) d w},
$$

where $\alpha_{v}(w)$ is a positive continuous density on $\mathbb{R}^{2}, d w$ denotes Lebesgue measure, and we assume $\int_{\mathbb{R}^{2}} \psi(w) \alpha_{v}(w) d w \neq 0$. Once again, this result is based on a precise asymptotics established for the expression $\sum_{\|\gamma\| \leq T} \phi(v \gamma)$ in Theorem 12.2 of [GW07], from which the ratio equidistribution theorem is an immediate corollary.

Let us note that the limiting density in the ratio theorem depends non-trivially on the starting point $v \in \mathbb{R}^{2}$, and for any given $v$, depends non-trivially on the 
norm. This is shown explicitly in [GW07], which in turn generalizes previous work of Ledrappier [L99], [L01] and Ledrappier-Pollicott [LP03], [LP05].

2.1.3. Lattice action on the quadratic surface (de-Sitter space). Consider now the homogeneous space $X=A \backslash \mathrm{SL}_{2}(\mathbb{R})$, where $A$ is the diagonal group. It can be realized as the one-sheeted hyperboloid in $\mathbb{R}^{3}$ given by the level set of a quadratic form of signature $(2,1)$ invariant under $\mathrm{SL}_{2}(\mathbb{R}) \cong \mathrm{SO}^{0}(2,1)$. The quadratic surface in question is also called de-Sitter space. Fix any norm on $M_{2}(\mathbb{R})$, and recall the polar coordinate system on de-Sitter's space, namely $\mathbb{R} \times S^{1} \mapsto X:(r, \omega) \mapsto$ $(\omega \cosh r, \sinh r)$. Then for every $\phi, \psi \in L^{1}(X)$ with compact support such that $\int_{X} \psi(r, \omega) \cosh r d r d \omega \neq 0$, and for almost every $v \in X$,

$$
\lim _{T \rightarrow \infty} \frac{\sum_{\|\gamma\|<T} \phi(v \gamma)}{\sum_{\|\gamma\|<T} \psi(v \gamma)}=\frac{\int_{X} \phi(r, \omega) \cosh r d r d \omega}{\int_{X} \psi(r, \omega) \cosh r d r d \omega} .
$$

Note that the limiting density here is the $G$-invariant measure on the quadratic surface, and is independent of the starting point and the norm. However this is not case for higher dimensional quadratic surfaces, see [GN14].

Once again, this result is based on a precise asymptotics, established in this case in [GN14], §1.5:

$$
\lim _{t \rightarrow \infty} \frac{1}{t} \sum_{\gamma \in \Gamma_{t}} \phi(v \gamma)=c_{2}(\Gamma) \int_{X} \phi(r, \omega)(\cosh r) d r d \omega
$$

where $\Gamma_{t}=\{\gamma \in \Gamma ; \log \|\gamma\| \leq t\}$, and $c_{2}(\Gamma)>0$. Further examples of precise asymptotics for actions of lattices in algebraic groups acting on homogeneous spaces with infinite invariant measure were established in [GW07], and [GN14]. These give rise to further ratio equidistribution theorems, or almost sure ratio ergodic theorems. A further discussion can be found in [GN12].

2.1.4. A ratio ergodic theorem for hyperbolic groups. We mention another recent (weighted) ratio ergodic theorem for actions of word-hyperbolic groups preserving a $\sigma$-finite measure $(X, \mu)$, established by Pollicott and Sharp [PS13]. Let $S$ be a symmetric generating set of such a group $\Gamma$, and let $S_{n}=\left\{\gamma \in \Gamma ;|\gamma|_{S}=n\right\}$, where $|\gamma|_{S}$ is the word length of $\gamma$ w.r.t. $S$. Then there exists $\rho=\rho_{S}>1$ such that for any $f, g \in L^{1}(X, \mu)$ with $g>0$ :

$$
\frac{\sum_{n=0}^{N} \rho^{-n} \sum_{|\gamma|_{S}=n} f(\gamma x)}{\sum_{n=0}^{N} \rho^{-n} \sum_{|\gamma|_{S}=n} g(\gamma x)}
$$

converges pointwise almost surely as $N \rightarrow \infty$. In the case that the group in question is the fundamental group of a closed surface of constant negative curvature, and the set of generators is the standard one, the limit is $\int_{X} f d \mu / \int_{X} g d \mu$ provided that the action is ergodic. 
2.1.5. Counterexample: balls on the free group (added in proof). A natural general question that can be posed in this context is the following. Let $\mathbb{F}=\left\langle a_{1}, \ldots, a_{r}\right\rangle$ be a rank $r \geq 2$ free group. Let $\left(T^{g}\right)_{g \in \mathbb{F}}$ be a conservative action on a standard $\sigma$-finite measure space $(X, \mathscr{B}, \lambda)$ by non-singular transformations. Let $\left(\hat{T}^{g}\right)_{g \in \mathbb{F}}$ be the induced isometric action on $L^{1}(X, \lambda)$, the Banach pre-dual of $L^{\infty}(X, \lambda)$. For $g \in \mathbb{F}$, let $|g|$ denote its word length with respect to the given generators. For $u, v \in L^{1}(X, \lambda)$ with $v>0$ let

$$
\operatorname{RATIO}_{n}[u, v]:=\frac{\sum_{|g| \leq n} \widehat{T}^{g} u}{\sum_{|g| \leq n} \widehat{T}^{k} v} .
$$

Does the sequence $\operatorname{RATIO}_{2 n}[u, v]$ converge pointwise almost everywhere as $n \rightarrow \infty$ ? This question was very recently answered negatively by Hochman in [Ho13], who constructed examples of measure preserving ergodic actions where the ratio ergodic theorem fails along (subsequences of ) the sequence of balls. The discussion in [Ho13] is very general and shows that such counterexamples exist whenever the sequence of balls in a finitely generated group fails to satisfy the Besicovich covering property. Thus this property is inextricably linked to the validity of the ratio ergodic theorem.

2.2. Statement of the main result. The main goal of the present paper is to give an exposition of a new dynamical approach to establishing ratio ergodic theorems for actions of free groups. This approach is motivated by the recent proof of the pointwise ergodic theorem for spherical averages in free groups developed in [BN13a]. We remark however that the method of proof we will develop is by no means restricted to free groups, and applies in much greater generality, see [BN13b] and [BN]. We will comment on these generalizations further below, but in the interest of simplicity of exposition will focus here on the free group only.

To set the notation, let $\mathbb{F}=\left\langle a_{1}, \ldots, a_{r}\right\rangle$ denote the free group of rank $r \geq 2$. Let $S=\bigcup_{i=1}^{r}\left\{a_{i}, a_{i}^{-1}\right\}$ be a free symmetric generating set. The reduced form of an element $g \in \mathbb{F}$ is the unique expression of the form $g=s_{1} \cdots s_{n}$ with $s_{i} \in S$ and $s_{i+1} \neq s_{i}^{-1}$ for all $i$. Define $|g|:=n$, the length of the reduced form of $g$. Define a distance on $\mathbb{F}$ by $d\left(g_{1}, g_{2}\right):=\left|g_{1}^{-1} g_{2}\right|$.

The boundary $\partial \mathbb{F}$ is the subspace of all sequences $\xi=\left(s_{1}, s_{2}, \ldots\right) \in S^{\mathbb{N}}$ such that $s_{i+1} \neq s_{i}^{-1}$ for all $i \geq 1$. It is naturally endowed with a Markov probability measure $v$ determined as follows. Let $\left(s_{1}, \ldots, s_{n}\right) \in S^{n}$ be an arbitrary sequence such that $s_{i+1} \neq s_{i}^{-1}$ for all $i \geq 1$. Then

$$
v\left(\left\{\xi \in \partial \mathbb{F}: \xi_{i}=s_{i} \text { for all } 1 \leq i \leq n\right\}\right)=(2 r)^{-1}(2 r-1)^{-(n-1)} .
$$

There is a natural action of $\mathbb{F}$ on $\partial \mathbb{F}$ by

$$
\left(t_{1} \cdots t_{n}\right)\left(s_{1}, s_{2}, \ldots\right)=\left(t_{1}, \ldots, t_{n-k}, s_{k+1}, s_{k+2}, \ldots\right)
$$

where $t_{1}, \ldots, t_{n} \in S, g=t_{1} \cdots t_{n} \in \mathbb{F}$ is in reduced form and $k$ is the largest number $\leq n$ such that $s_{i}^{-1}=t_{n+1-i}$ for all $i \leq k$. 
The action preserves the measure class of $\nu$. For any $\xi \in \partial \mathbb{F}$, the set

$$
H_{\xi}=\left\{g \in \mathbb{F}: \frac{d \nu \circ g^{-1}}{d v}(\xi)=1\right\}
$$

is the horosphere centered at $\xi$ passing through the identity element $e$. The horosphere can alternatively be described as the set of all elements $g \in \mathbb{F}$ such that for some $n=n(g)>0,\left(g^{-1} \xi\right)_{i}=\xi_{i}$ for all $i>n$ (i.e., $g^{-1}$ preserves the 'tail' of $\xi$, from some point onwards, or equivalently, $|g|=2 n$ and precisely the first $n$ letters of $g$ coincide with those of $\xi$ ).

We let $\mathcal{R}_{0} \subset \partial \mathbb{F} \times \partial \mathbb{F}$ be the equivalence relation given by $\xi \sim \mathcal{R}_{0} \xi^{\prime}$ if and only if there is an $n>0$ such that $\xi_{i}=\xi_{i}^{\prime}$ for all $i>n$. In other words, $\xi \sim_{\mathcal{R}_{0}} \xi^{\prime}$ if there exists $g \in H_{\xi}$ such that $g^{-1} \xi=\xi^{\prime}$.

Now let $(X, \mathscr{B}, \lambda)$ be a standard $\sigma$-finite measure space on which $\mathbb{F}$ acts by nonsingular transformations. Let $\mathcal{R}_{0}(X) \subset X \times \partial \mathbb{F} \times X \times \partial \mathbb{F}$ be the equivalence relation under which $(x, \xi)$ is equivalent to $\left(y, \xi^{\prime}\right)$ if and only if there is a $g \in H_{\xi}$ such that $\left(y, \xi^{\prime}\right)=\left(g^{-1} x, g^{-1} \xi\right)$.

Our main theorem is:

Theorem 2.1. If $u, v \in L^{1}(X \times \partial \mathbb{F}, \lambda \times v)$, with $v>0$ almost everywhere, then the ratios

$$
\operatorname{RATIO}_{2 n}[u, v](x, \xi):=\frac{\sum_{g-1 \in H_{\xi},|g| \leq 2 n} \hat{T}^{g} u(x, \xi)}{\sum_{g-1 \in H_{\xi},|g| \leq 2 n} \hat{T}^{g} v(x, \xi)}
$$

converge pointwise almost everywhere as $n \rightarrow \infty$ to a $\mathcal{R}_{0}(X)$-invariant function $r(u, v)$.

Furthermore, if $\mathcal{R}_{0}(X)$ is ergodic then $r(u, v)=\frac{\int u d(\lambda \times v)}{\int v d(\lambda \times v)}$, and the conclusions hold for any choice of $v \in L^{1}(X \times \partial \mathbb{F})$ provided only that it satisfies $\int v d(\lambda \times v) \neq 0$.

It is an open question whether the analogous statement with the condition $|g| \leq 2 n$ replaced by $|g|=2 n$ holds true.

In the case when $(X, \mathcal{B}, \lambda)$ is a probability space and the action is measurepreserving, we proved in [BN13a] that the averages

$$
\frac{1}{\#\left\{g^{-1} \in H_{\xi},|g| \leq 2 n\right\}} \sum_{g^{-1} \in H_{\xi},|g| \leq 2 n} \hat{T}^{g} u(x, \xi)
$$

converge almost everywhere to $\mathbb{E}_{\lambda \times \nu}\left[u \mid \mathcal{R}_{0}(X)\right](x, \xi)$ which is the conditional expectation of $u$ on the $\sigma$-algebra of $\mathcal{R}_{0}(X)$-saturated sets. Moreover, if $u(x, \xi)=u(x)$ is a function of the first argument only then $\mathbb{E}_{\lambda \times v}\left[u \mid \mathcal{R}_{0}(X)\right](x, \xi)$ is equal to $\mathbb{E}_{\lambda}\left[u \mid \mathbb{F}^{2}\right]$, the conditional expectation of $u$ on the $\sigma$-algebra of $\mathbb{F}^{2}$-invariant sets (where $\mathbb{F}^{2}<\mathbb{F}$ is the index 2 subgroup consisting of all $g \in \mathbb{F}$ with $|g|$ even). In particular if $\mathbb{F}^{2}$ acts ergodically on $(X, \mathscr{B}, \lambda)$ then the above averages converge pointwise a.e. to the 
integral $\int u(x) d \lambda$. By averaging the above averages over the whole boundary it is possible to establish the pointwise ergodic theorem for uniform spherical averages in free groups, as well as for many other averages [BN13a].

In the general case, when $(X, \mathcal{B}, \lambda)$ is $\sigma$-finite and the action is merely nonsingular then there are examples (provided in $\S 5$ ) showing that $\mathbb{E}_{\lambda \times v}\left[u \mid \mathcal{R}_{0}(X)\right]$ does not necessarily equal $\mathbb{E}_{\lambda}\left[u \mid \mathbb{F}^{2}\right]$. Many interesting cases remain open. For example, it is not known whether $\mathbb{E}_{\lambda \times v}\left[u \mid \mathcal{R}_{0}(X)\right]=\mathbb{E}_{\lambda}\left[u \mid \mathbb{F}^{2}\right]$ in the special case when $X$ is the isometry group of Euclidean $n$-space, $\phi: \mathbb{F} \rightarrow X$ is a homomorphism onto a dense subgroup and the action $\left(T^{g}\right)_{g \in \mathbb{F}}$ is given by $T^{g} x=\phi(g) x$.

2.3. Organization of the paper. To begin, in $\S 3$ we prove a ratio ergodic theorem for measured equivalence relations with respect to averages on subsets satisfying some extreme invariance properties. It seems likely that the hypotheses in this result can be relaxed. Next we show in $\S 4$ that the horospherical equivalence relation described above have natural subsets that satisfy the hypotheses of $\S 3$. This implies our main result for the free group. In $\S 5$ we exhibit some examples pertaining to the issue of whether ergodicity of $\mathbb{F} \curvearrowright(X, \mathcal{B}, \lambda)$ implies ergodicity of the relation $\mathcal{R}_{0}(X)$.

Acknowledgements. The authors would like to thank Mike Hochman for useful conversations, and the (anonymous) referees for useful comments.

\section{A ratio ergodic theorem for amenable equivalence relations}

Consider a standard $\sigma$-finite measure space $(B, \nu)$ with a Borel equivalence relation $\mathcal{R} \subset B \times B$. We assume that $\mathcal{R}$ is discrete and that the measure $v$ is non-singular w.r.t. $\mathcal{R}$ (or $\mathcal{R}$-quasi-invariant in equivalent terminology). Discreteness of the relation $\mathcal{R}$ means that for almost every $b \in B$, the equivalence class $[b]$ of $b$ is at most countable. Quasi-invariance of the measure under the relation means that for any Borel $A \subset B$ with $v(A)=0$, its saturation $[A]:=\bigcup_{a \in A}[a]$ also has $v$-measure zero. Integrating the counting measures on the fibers of the left projection $(x, y) \in$ $\mathcal{R} \mapsto x$ gives the left counting measure $M$ on $\mathcal{R}$ satisfying $d M(x, y)=d \nu(x)$. The right counting measure $\check{M}$ is defined by $d \check{M}(x, y)=d M(y, x)$. Because $v$ is quasi-invariant, these two measures are equivalent and the Radon-Nikodym cocycle is defined by $D(x, y) d \check{M}(x, y):=d M(x, y)$. Thus the cocycle identity $D(x, z)=$ $D(x, y) D(y, z)$ is satisfied, for almost all $x, y, z \in B$..

Suppose that $\mathscr{F}=\left\{\mathscr{F}_{n}\right\}_{n=1}^{\infty}$ is a sequence of measurable functions $\mathscr{F}_{n}: B \rightarrow 2_{\text {fin }}^{B}$ (where $2_{\text {fin }}^{B}$ denotes the space of finite subsets of $B$ ) such that $\mathscr{F}_{n}(b)$ is a subset of the $\mathcal{R}$-equivalence class of $b$ for almost every $b$. We will need the following definitions.

(1) Let $\operatorname{Inn}(\mathcal{R})$ denote the group of inner automorphisms of $\mathcal{R}$ (also known as the full group of $\mathcal{R}$ ). These are invertible measurable maps $\phi: B \rightarrow B$ with graph contained in $\mathcal{R}$ (up to a null set). A set $\Phi \subset \operatorname{Inn}(\mathcal{R})$ generates $\mathcal{R}$ if for 
almost every $\left(b_{1}, b_{2}\right) \in \mathcal{R}$ there exists $\phi \in\langle\Phi\rangle$ such that $\phi\left(b_{1}\right)=b_{2}$ (where $\langle\Phi\rangle$ denotes the group generated by $\Phi)$.

(2) $\mathcal{F}$ is extremely asymptotically invariant if $\lim _{n \rightarrow \infty}\left|\mathscr{F}_{n}(b)\right|=+\infty$ for $v$-a.e. $b$ and there exists a countable generating set $\Phi \subset \operatorname{Inn}(\mathcal{R})$ such that for every $\phi \in \Phi$ and almost every $b \in B$ there exists an $N=N(\phi, b)$ such that $n>N$ implies $\widetilde{F}_{n}(b)=\phi\left(\widetilde{F}_{n}(b)\right)$.

(3) $\mathcal{F}$ satisfies the extreme Besicovich property if for almost every $\left(b, b^{\prime}\right) \in \mathcal{R}$ and any $n \geq 0$, either $\mathcal{F}_{n}(b)=\mathscr{F}_{n}\left(b^{\prime}\right)$ or $\mathscr{F}_{n}(b) \cap \mathscr{F}_{n}\left(b^{\prime}\right)=\emptyset$. Moreover, we require that there exists an $N=N\left(b, b^{\prime}\right)$ such that $n \geq N$ implies $\mathscr{F}_{n}(b)=$ $\mathcal{F}_{n}\left(b^{\prime}\right)$.

(4) $\mathcal{F}$ is anchored if $b \in \mathscr{F}_{n}(b)$ for every $n$ and almost every $b$.

Remark 3.1. Consider a non-singular hyperfinite relation $\mathcal{T}$ on $(B, v)$, namely a relation which can be represented as an increasing union $\mathcal{T}=\bigcup_{n \in \mathbb{N}} \mathcal{T}_{n}$ of equivalence relations $\mathcal{T}_{n}$ with finite classes. Clearly, $\mathcal{T}$ is anchored, and satisfies the extreme Besicovich property w.r.t. to the choice of $\mathcal{F}_{n}(b)=\mathcal{T}_{n}(b)$, where $\mathcal{T}_{n}(b)$ is the finite $\mathcal{T}_{n}$-equivalence class of $b \in B$. Furthermore, $\mathcal{T}$ is clearly extremely asymptotically invariant, where for $\Phi$ we choose the group generated by all the inner automorphisms of the relations $\mathcal{T}_{n}, n \in \mathbb{N}$. Since every discrete non-singular amenable Borel equivalence relation is in fact hyperfinite, the ratio ergodic theorem stated below applies in great generality.

For $u, v \in L^{p}(B, v)$ with $v>0$ on $B$, consider the sums $\operatorname{SUM}_{n}^{\mathcal{F}}[u] \in L^{p}(B)$ and ratios $\operatorname{RATIO}_{n}^{\mathscr{F}}[u, v]$ defined by

$$
\begin{aligned}
\operatorname{SUM}_{n}^{\mathcal{F}}[u](b) & :=\sum_{b^{\prime} \in \mathcal{F}_{n}(b)} u\left(b^{\prime}\right) D\left(b^{\prime}, b\right), \\
\operatorname{RATIO}_{n}^{\mathcal{F}}[u, v](b) & :=\frac{\operatorname{SUM}_{n}^{\mathcal{F}}[u](b)}{\operatorname{SUM}_{n}^{\mathcal{F}}[v](b)} .
\end{aligned}
$$

For $u \in L^{1}(B, v)$ let $\mathbb{E}_{v}[u \mid \mathcal{R}]$ denotes the conditional expectation of $u$ on the $\sigma$ algebra of $\mathcal{R}$-invariant sets with respect to the measure $v$.

The main result of this section is:

Theorem 3.1. If $\mathcal{F}$ is extremely asymptotically invariant, anchored and satisfies the extreme Besicovich property then $\mathcal{F}$ is a pointwise ratio ergodic sequence in $L^{1}$. Namely, for every $u, v \in L^{1}(B, v)$, with $v>0$ on $B, \operatorname{RATIO}_{n}^{\mathcal{F}}[u, v]$ converges pointwise almost everywhere as $n \rightarrow \infty$ to a function $r(u, v)$ on $B$ satisfying

- $r(u, v)$ is an $\mathcal{R}$-invariant function,

- $\int f \cdot r(u, v) v d v=\int f \cdot u d v$ for any $f \in L^{\infty}(B, v)$ which is $\mathcal{R}$-invariant. 
In general

$$
r(u, v)=\mathbb{E}_{v_{v}}\left[\frac{u}{v} \mid \mathcal{R}\right]
$$

is the conditional expectation of $\frac{u}{v}$ on the $\sigma$-algebra of $\mathcal{R}$-invariant sets, with respect to the measure $\nu_{v}$ defined by $d v_{v}=v d v$.

In particular, if $\mathcal{R}$ is ergodic then $r(u, v)$ equals the constant $\frac{\int u d v}{\int v d v}$ almost everywhere. In that case, the conclusions hold for any choice of $v \in L^{1}(B, v)$ provided only that $\int_{B} v d v \neq 0$.

We would like to point out that the hypotheses are certainly not necessary. For example, the classical ratio ergodic theorem for averages along intervals of $\mathbb{Z}$ could be phrased in the language of equivalence relations. But expanding intervals in $\mathbb{Z}$ are neither extremely asymptotically invariant nor do they satisfy the extreme Besicovich property. It is an open problem to determine general hypotheses on $\mathscr{F}$ guaranteeing that it is a ratio ergodic sequence.

The proof of Theorem 3.1 follows a classical recipe: we first prove that it holds true for fixed $v$ and for $u$ in a naturally defined dense subset of $L^{1}$. Then with the aid of a maximal inequality, we show that it holds for all $u$ in $L^{1}$. To be precise:

Theorem 3.2 (Dense set of good functions). If $\widetilde{F}$ is extremely asymptotically invariant then, given $v \in L^{1}(B, v)$ with $v>0$ on $B$, there exists a norm dense subspace $\mathscr{L}_{v} \subset L^{1}(B, v)$ such that for all $u \in \mathscr{L}_{v}, \mathrm{RATIO}_{n}^{\mathcal{F}}[u, v]$ converges pointwise almost everywhere.

Let $M^{\mathscr{F}}[u, v]:=\sup _{n}\left|\operatorname{RATIO}_{n}^{\mathscr{F}}[u, v]\right|$. We will prove the following weak-type $(1,1)$ maximal inequality.

Theorem 3.3 ( $L^{1}$ maximal inequality). Suppose that $\mathcal{F}$ is anchored and satisfies the extreme Besicovich property. Then for any $u, v \in L^{1}(B, v)$ with $v>0$ on $B$, and any $\epsilon>0$,

$$
v_{v}\left(\left\{b \in B: M^{\mathcal{F}}[u, v](b) \geq \epsilon\right\}\right) \leq \frac{1}{\epsilon} \int_{\left\{M^{\mathcal{F}}[u, v](b)>\epsilon\right\}} u(b) d v(b) \leq \frac{\|u\|_{L^{1}(B, v)}}{\epsilon}
$$

where $v_{v}$ is the measure $d v_{v}=v d v$.

Finally, when $\mathcal{R}$ is ergodic, the proof of the last statement in Theorem 3.1 follows from the observation that given $u, v \in L^{1}(B, v)$, with $\int_{B} v d v \neq 0$, we can choose $v_{0} \in L^{1}(B, v)$, with $v_{0}>0$, and then consider the ratios $\operatorname{RATIO}_{n}^{\mathcal{F}}\left[u, v_{0}\right]$ and $\operatorname{RATIO}_{n}^{\mathcal{F}}\left[v, v_{0}\right]$. Both are well defined, and for almost every $b \in B$, the second is eventually non-zero, and converges to the non-zero constant $\int v d v / \int v_{0} d v$. Hence the ratio of the two ratios converges almost surely to $\int u d v / \int v d v$, which is the final conclusion stated in Theorem 3.1. 


\subsection{A dense set of good functions}

Lemma 3.2. For any $f \in L^{1}(B, v)$ and $\phi \in \operatorname{Inn}(\mathcal{R})$,

$$
\int f(b) D(\phi b, b) d v(b)=\int f\left(\phi^{-1} b\right) d v(b) .
$$

Proof. Recall that $D(\phi b, b) d \check{M}(\phi b, b)=d M(\phi b, b)$. Therefore

$$
\begin{aligned}
\int f(b) D(\phi b, b) d v(b) & =\int f(b) D(\phi b, b) d \check{M}(\phi b, b) \\
& =\int f(b) d M(\phi b, b)=\int f\left(\phi^{-1} b\right) d v(b) .
\end{aligned}
$$

Lemma 3.3. Let $\mathcal{F}$ be extremely asymptotically invariant and $\Phi \subset \operatorname{Inn}(\mathcal{R})$ be a countable generating set witnessing its extreme asymptotic invariance. For $\phi \in \Phi$ and $u \in L^{1}(B, v)$, define $u_{\phi} \in L^{1}(B, v)$ by

$$
u_{\phi}(b):=u(b)-u(\phi(b)) D(\phi(b), b) .
$$

Then $\mathrm{SUM}_{n}^{\mathcal{F}}\left[u_{\phi}\right]$ converges pointwise almost everywhere to $0=\mathbb{E}_{v}\left[u_{\phi} \mid \mathcal{R}\right]$. Moreover the span of $\left\{u_{\phi}: u \in L^{1}(B, v), \phi \in \Phi\right\}$ is norm dense in $L_{0}^{1}(B)$ (= the set of $w \in L^{1}(B, v)$ with $\left.\mathbb{E}_{v}[w \mid \mathcal{R}]=0\right)$.

Proof. Note that

$$
\operatorname{SUM}_{n}^{\mathcal{F}}\left[u_{\phi}\right](b)=\sum_{b^{\prime} \in \mathcal{F}_{n}(b)} u\left(b^{\prime}\right) D\left(b^{\prime}, b\right)-u\left(\phi\left(b^{\prime}\right)\right) D\left(\phi\left(b^{\prime}\right), b\right)=0
$$

for all $n>N(\phi, b)$ since $\phi\left(\mathscr{F}_{n}(b)\right)=\mathscr{F}_{n}(b)$. In particular $\operatorname{SUM}_{n}^{\mathcal{F}}\left[u_{\phi}\right]$ converges pointwise almost everywhere to 0 . By Lemma $3.2, \mathbb{E}_{v}\left[u_{\phi} \mid \mathcal{R}\right]=0$. To see that the span of the set of all functions of the above form is norm dense in $L_{0}^{1}(B)$, let $L_{0}^{\infty}(B)$ be the Banach dual of $L_{0}^{1}(B)$. Suppose $f \in L_{0}^{\infty}(B), \int f u_{\phi} d v=0$ for all $u \in L_{0}^{1}(B)$ and $\phi \in \Phi$. Then

$$
\int f(b) u(b) d v(b)=\int f(b) u(\phi(b)) D(\phi(b), b) d v(b)=\int f\left(\phi^{-1} b\right) u(b) d v(b)
$$

by the previous lemma. Since this equality holds for all $u \in L_{0}^{1}(B)$, it follows that $f=f \circ \phi^{-1}$ a.e. for every $\phi \in \Phi$. Since $\Phi$ is countable and generating, this implies that $f$ is $\mathcal{R}$-invariant. So $\int_{B} f u d v=\int_{B} f \mathbb{E}_{v}(u) d v=0$ for all $u \in L_{0}^{1}(B)$, and $f$ defines the zero functional. This proves that the weak closure of the span of the collection of functions of the form $u_{\phi}$ (with $u \in L^{1}(B, v)$ and $\phi \in \Phi$ ) is all of $L_{0}^{1}(B)$. Because the weak closure of a subspace equals its norm closure, this proves the lemma. 
We will now use these facts in order to construct, given $v \in L^{1}(B, v)$, a normdense subset $\mathscr{L}_{v} \subset L^{1}(B, v)$, such that for $u \in \mathscr{L}_{v}$, $\frac{\operatorname{SUM}_{n}^{\mathscr{F}}[u]}{\operatorname{SUM}_{n}^{\mathscr{F}}[v]}$ converges almost everywhere.

Proof of Theorem 3.2. Consider the linear subspace $\mathscr{L}_{v}$ of $L^{1}(B, v)$ spanned by all functions of the form $w v$, for all bounded functions $w$ which are $\mathcal{R}$-invariant, together with the span of all the functions of the form $u_{\phi}$, as $\phi$ ranges over the set $\Phi$ of inner automorphisms defined above, and $u$ over $\left(L^{1} \cap L^{\infty}\right)(B, v)$. Thus

$$
\begin{gathered}
\mathscr{L}_{v}=\left\{w v+\sum_{i \in I}\left(u_{i}\right)_{\phi_{i}} ; w \in L^{\infty}(B, v)^{\mathcal{R}}, u_{i} \in\left(L^{1} \cap L^{\infty}\right)(B, v),\right. \\
\left.\phi_{i} \in \Phi,|I|<\infty\right\} .
\end{gathered}
$$

Since $w$ is $\mathcal{R}$-invariant, clearly $\operatorname{RATIO}_{n}^{\mathscr{F}}[w v, v]=w$. The proof of Lemma 3.3 implies that for every function $y \in \mathscr{L}_{v}$ the ratios $\operatorname{RATIO}_{n}^{\mathscr{F}}[y, v]$ converge almost everywhere. To show that $\mathscr{L}_{v}$ is norm dense in $L^{1}(B, v)$, assume that $k \in L^{\infty}(B, v)$ satisfies $\int_{B} k y d v=0$ for all $y \in \mathscr{L}_{v}$, and we will show that $k=0$. But under the foregoing condition we have for $y=u_{\phi}$ in particular that

$$
\int k(b) u(b) d v(b)=\int k(b) u(\phi(b)) D(\phi(b), b) d v(b)=\int k\left(\phi^{-1} b\right) u(b) d v(b) .
$$

for every $u \in L^{1}(B, v)$ and $\phi \in \Phi$ by Lemma 3.2. So $k$ must be invariant under $\Phi$. As we noted in the proof of Lemma 3.3 above, it follows that $k$ is an $\mathcal{R}$-invariant function. Therefore by definition $k v \in \mathscr{L}_{v}$, and hence $\int_{B} k \cdot k v d v=0$, so that $k$ vanishes on the support of $v$ which is all of $B$. Thus $\mathscr{L}_{v}$ is weakly dense and therefore norm dense in $L^{1}(B, v)$. This concludes the proof of Theorem 3.2.

3.2. Identifying the limit. We proceed to identify the limit of $\operatorname{RATIO}_{n}^{\mathcal{F}}[u, v]$ as the $\mathcal{R}$-invariant function given by the conditional expectation $\mathbb{E}_{\nu_{v}}\left[\frac{u}{v} \mid \mathcal{R}\right]$, where $d \nu_{v}=$ $v d v$.

Lemma 3.4. Let $\mathscr{L}_{v}$ be the subspace defined in the proof of Theorem 3.2. Then for every $y \in \mathscr{L}_{v}$,

$$
\lim _{n \rightarrow \infty} \operatorname{RATIO}_{n}^{\mathcal{F}}[y, v]=\mathbb{E}_{v_{v}}\left[\frac{y}{v} \mid \mathcal{R}\right] .
$$

Proof. First, we already saw in the proof of Theorem 3.2 that if $w v+\sum_{i \in I}\left(u_{i}\right)_{\phi_{i}}=$ $y \in \mathscr{L}_{v}$ with $w$ an $\mathcal{R}$-invariant function, then $\lim _{n \rightarrow \infty} \operatorname{RATIO}_{n}^{\mathcal{F}}[y, v]=w$. If $y$ has another representation $y^{\prime}=w^{\prime} v+\sum_{j \in J}\left(u_{j}^{\prime}\right)_{\phi_{j}^{\prime}}$, then it follows that $w=w^{\prime}$, so that $w$ is uniquely determined by $y$.

Thus we can consider the well-defined map $y=w v+\sum_{i \in I}\left(u_{i}\right)_{\phi_{i}} \mapsto w v=$ $\Psi_{v}(y)$. We claim that $\Psi_{v}: \mathscr{L}_{v} \rightarrow L^{1}(B, v)$ is a contraction when taking the 
$L^{1}(B, v)$-norm on both sides. Indeed, for any bounded $\mathcal{R}$-invariant function $k$, we have

$$
\int_{B} k y d v=\int_{B}\left(k w v+k \sum_{i \in I}\left(u_{i}\right)_{\phi_{i}}\right) d v=\int_{B} k w v d v
$$

Taking $k=\operatorname{sign}(w), k$ is obviously $\mathcal{R}$-invariant since $w$ is, and thus

$$
\begin{aligned}
\left\|\Psi_{v}(y)\right\|_{L^{1}(B, v)} & =\|w v\|_{L^{1}(B, v)}=\int_{B}|w| v d v \\
& =\int_{B} \operatorname{sign}(w) w v d v=\int_{B} \operatorname{sign}(w) y d v \leq\|y\|_{L^{1}(B, v)} .
\end{aligned}
$$

It follows that $\Psi_{v}: \mathscr{L}_{v} \rightarrow L^{1}(B, v)$ can be extended to a linear operator of norm bounded by 1 from the closure of $\mathscr{L}_{v}$, namely $L^{1}(B, v)$, to $L^{1}(B, v)$.

Clearly, the foregoing shows that for every $y \in \mathscr{L}_{v}, \Psi_{v}(y)=w v$ has the property that for every bounded $\mathcal{R}$-invariant function $k$,

$$
\int_{B} k \Psi_{v}(y) d v=\int_{B} k y d v .
$$

By the norm density of $\mathscr{L}_{v}$ and by continuity of $\Psi_{v}(y)$, it follows that the last equation is valid also for $\Psi_{v}(z)$ for every $z \in L^{1}(B, v)$.

We now claim that for every $y \in \mathscr{L}_{v}$,

$$
\lim _{n \rightarrow \infty} \operatorname{RATIO}_{n}^{\mathscr{F}}[y, v]=w=\frac{\Psi_{v}(y)}{v}=\mathbb{E}_{v_{v}}\left[\frac{y}{v} \mid \mathcal{R}\right] .
$$

Indeed the convergence of the ratios to $w$ was established above, and the fact that $w$ is the stated conditional expectation is equivalent to

$$
\int_{B} k w \cdot v d v=\int_{B} k \frac{y}{v} \cdot v d v
$$

for every $k \in L^{\infty}(B, v)^{\mathcal{R}}$, which was just verified.

In order to extend the convergence result stated in Lemma 3.4 to an arbitrary function $u \in L^{1}(B, v)$, we will use the following facts.

First note that since $\mathscr{L}_{v}$ is norm dense in $L^{1}(B, v)$, the range of $\Psi_{v}: L^{1}(B, v) \rightarrow$ $L^{1}(B, v)$ is contained in the closed subspace of $L^{1}(B, v)$ consisting of functions of the form $w v$, where $w$ is an $\mathcal{R}$-invariant function. Indeed $w_{n} v$ is a Cauchy sequence in $L^{1}(B, v)$ if and only if $w_{n}$ is a Cauchy sequence in $L^{1}\left(B, v_{v}\right)$, and in fact the space $\left\{w v \in L^{1}(B, v), w\right.$ is $\mathcal{R}$ invariant $\}$ is clearly isometrically isomorphic with the Banach space of $\mathcal{R}$-invariant functions in $L^{1}\left(B, v_{v}\right)$. Hence if $y_{n} \in \mathscr{L}_{v}$ and $y_{n} \rightarrow u, \Psi_{v}\left(y_{n}\right)=w_{n} v \rightarrow z$ in $L^{1}(B, v)$, then $w_{n} \rightarrow \frac{z}{v}$ in $L^{1}\left(B, v_{v}\right), w=\frac{z}{v}$ is $\mathcal{R}$-invariant, and $\Psi_{v}(u)=w v$. 
Thus $\Psi_{v}(u)=w(u) v$, with $w(u)$ a uniquely determined $\mathcal{R}$-invariant function. Clearly, the composition $u \mapsto \Psi_{v}(u) \mapsto \frac{\Psi_{v}(u)}{v}=w(u)$ is a bounded linear map from $L^{1}(B, v)$ to $L^{1}\left(B, v_{v}\right)$, with norm bounded by 1 .

Furthermore, we claim that

$$
\frac{\Psi_{v}(u)}{v}=w(u)=\mathbb{E}_{v_{v}}\left[\frac{u}{v} \mid \mathcal{R}\right]
$$

for every $u \in L^{1}(B, v)$. This follows because the conditional expectation is a bounded linear operator on $L^{1}\left(B, v_{v}\right)$ with closed range, $\mathscr{L}_{v}$ is norm-dense in $L^{1}(B, v)$, and these identities hold for $u \in \mathscr{L}_{v}$, as we saw in the proof of Lemma 3.4.

Finally, we can therefore conclude that the composition $u \mapsto \mathbb{E}_{v_{v}}\left[\frac{u}{v} \mid \mathcal{R}\right]=w(u)$ is a bounded linear map from $L^{1}(B, v)$ to $L^{1}\left(B, v_{v}\right)^{\mathcal{R}}$, with norm at most 1 .

We will now show that the property that $\lim _{n \rightarrow \infty} \operatorname{RATIO}_{n}^{\mathcal{F}}[u, v]=\mathbb{E}_{v_{v}}\left[\frac{u}{v} \mid \mathcal{R}\right]$ extends to all $u \in L^{1}(B, v)$, by an argument that employs the weak-type $(1,1)$ ratio maximal inequality.

\subsection{Applying the ratio maximal inequality}

Proof of Theorem 3.1 assuming Theorem 3.3. Let $u$ be any function in $L^{1}(B, v)$, fix $\epsilon>0$ and $y \in \mathscr{L}_{v}$ which satisfies $\|u-y\|_{L^{1}(B, v)}<\epsilon^{2}$. have

Applying the fact that $\lim _{n \rightarrow \infty} \operatorname{RATIO}_{n}^{\mathcal{F}}[y, v]=\mathbb{E}_{v_{v}}\left[\frac{y}{v} \mid \mathcal{R}\right]$ almost surely, we

$$
\begin{aligned}
\limsup _{n \rightarrow \infty} & \left|\operatorname{RATIO}_{n}^{\mathcal{F}}(u, v)-\mathbb{E}_{v_{v}}\left[\frac{u}{v} \mid \mathcal{R}\right]\right| \\
\leq & \limsup _{n \rightarrow \infty}\left|\operatorname{RATIO}_{n}^{\mathcal{F}}(u-y, v)\right| \\
& \quad+\limsup _{n \rightarrow \infty}\left|\operatorname{RATIO}_{n}^{\mathcal{F}}(y, v)-\mathbb{E}_{v_{v}}\left[\frac{y}{v} \mid \mathcal{R}\right]\right|+\left|\mathbb{E}_{v_{v}}\left[\frac{u-y}{v} \mid \mathcal{R}\right]\right| \\
\quad & \limsup _{n \rightarrow \infty}\left|\operatorname{RATIO}_{n}^{\mathcal{F}}(u-y, v)\right|+\left|\mathbb{E}_{v_{v}}\left[\frac{u-y}{v} \mid \mathcal{R}\right]\right| .
\end{aligned}
$$

The first summand is estimated by the maximal inequality stated in Theorem 3.3:

$$
v_{v}\left\{\limsup _{n \rightarrow \infty}\left|\operatorname{RATIO}_{n}^{\mathcal{F}}(u-y, v)\right|>\epsilon\right\} \leq \frac{1}{\epsilon}\|u-y\|_{L^{1}(B, v)}<\epsilon .
$$

The second summand is estimated by the discussion preceding the proof:

$$
v_{v}\left\{\left|\mathbb{E}_{v_{v}}\left[\frac{u-y}{v} \mid \mathcal{R}\right]\right|>\epsilon\right\} \leq \frac{1}{\epsilon}\|w(u)-w(y)\|_{L^{1}\left(B, v_{v}\right)} \leq \frac{1}{\epsilon}\|u-y\|_{L^{1}(B, v)}<\epsilon
$$

Since these inequalities are valid for all $\epsilon>0$, we have

$$
\limsup _{n \rightarrow \infty}\left|\operatorname{RATIO}_{n}^{\mathcal{F}}(u, v)-\mathbb{E}_{v_{v}}\left[\frac{u}{v} \mid \mathcal{R}\right]\right|=0
$$


almost everywhere and this completes the proof of Theorem 3.1 subject to the proof of Theorem 3.3, to which we now turn.

\subsection{The weak-type ratio maximal inequality in $L^{1}$}

Proof of Theorem 3.3. Without loss of generality, we may assume that $u$ is nonnegative. For $T>0$, define $M_{T}^{\mathcal{F}}[u, v]: B \rightarrow \mathbb{R}$ by

$$
M_{T}^{\mathcal{F}}[u, v](b):=\sup _{T \geq n \geq 0} \operatorname{RATIO}_{n}^{\mathcal{F}}[u, v](b) .
$$

Let $\epsilon>0$. Let $D$ be the set of all $b \in B$ such that $M^{\mathscr{F}}[u, v](b)>\epsilon$ and $D_{T}$ be the set of all $b \in B$ such that $M_{T}^{\mathcal{F}}[u, v](b)>\epsilon$. Since $\left\{D_{T}\right\}_{T>0}$ is an increasing sequence with $D=\bigcup_{T>0} D_{T}$, it suffices to prove that $v_{v}\left(D_{T}\right) \leq \epsilon^{-1} \int_{D_{T}} u d v$ for each $T>0$.

Fix $T>0$ and let $\rho: D_{T} \rightarrow \mathbb{R}$ be defined by $\rho(b)=n$ if $n$ is the largest number such that $\operatorname{SUM}_{n}^{\mathcal{F}}[u, v](b)>\epsilon$ and $n \leq T$. Let $\mathcal{C}$ be the collection of all sets of the form $\mathcal{F}_{\rho(b)}(b)$ for $b \in D_{T}$. Let $E$ be the union of all sets in $\mathcal{C}$. Because $\mathcal{F}$ is anchored, it follows that $D_{T} \subset E$. Now the extreme Besicovich property of $\mathscr{F}$ implies $D_{T}=E$ and the sets in $\mathcal{C}$ are pairwise disjoint.

Define $F_{u}: B \times B \rightarrow \mathbb{R}$ by

- $F_{u}\left(b, b^{\prime}\right)=u(b)\left|\mathcal{F}_{\rho(b)}(b)\right|^{-1}$ if $b \in E$ and $b^{\prime} \in \mathcal{F}_{\rho(b)}(b)$;

- $F_{u}\left(b, b^{\prime}\right)=0$ otherwise.

Define $F_{v}$ similarly with $v$ in place of $u$.

For any $b, b^{\prime}$ either $\mathcal{F}_{\rho(b)}(b)=\mathscr{F}_{\rho\left(b^{\prime}\right)}\left(b^{\prime}\right)$ or $\mathcal{F}_{\rho(b)}(b) \cap \mathcal{F}_{\rho\left(b^{\prime}\right)}\left(b^{\prime}\right)=\emptyset$. Therefore,

$$
\begin{aligned}
\int_{E} u(b) d v(b) & =\int_{B} \sum_{b^{\prime}} F_{u}\left(b, b^{\prime}\right) d v(b) \\
& =\int_{\mathcal{R}} F_{u}\left(b, b^{\prime}\right) d M\left(b, b^{\prime}\right) \\
& =\int_{\mathcal{R}} F_{u}\left(b, b^{\prime}\right) D\left(b, b^{\prime}\right) d \check{M}\left(b, b^{\prime}\right) \\
& =\int_{B} \sum_{b} F_{u}\left(b, b^{\prime}\right) D\left(b, b^{\prime}\right) d v\left(b^{\prime}\right) \\
& =\int_{E} \frac{1}{\left|\mathscr{F}_{\rho\left(b^{\prime}\right)}\left(b^{\prime}\right)\right|} \sum_{b \in \mathcal{F}_{\rho\left(b^{\prime}\right)}\left(b^{\prime}\right)} u(b) D\left(b, b^{\prime}\right) d v\left(b^{\prime}\right) \\
& =\int_{E} \frac{1}{\left|\mathscr{F}_{\rho\left(b^{\prime}\right)}\left(b^{\prime}\right)\right|} \operatorname{SUM}_{\rho\left(b^{\prime}\right)}^{\mathcal{F}}[u]\left(b^{\prime}\right) d v\left(b^{\prime}\right) .
\end{aligned}
$$


Similarly,

$$
\int_{E} v(b) d v(b)=\int_{E} \frac{1}{\left|\mathcal{F}_{\rho\left(b^{\prime}\right)}\left(b^{\prime}\right)\right|} \operatorname{SUM}_{\rho\left(b^{\prime}\right)}^{\mathcal{F}}[v]\left(b^{\prime}\right) d v\left(b^{\prime}\right) .
$$

By definition of $D_{T}$,

$$
\begin{aligned}
\int_{D_{T}} & \frac{1}{\left|\mathcal{F}_{\rho\left(b^{\prime}\right)}\left(b^{\prime}\right)\right|} \operatorname{SUM}_{\rho\left(b^{\prime}\right)}^{\mathcal{F}}[u]\left(b^{\prime}\right) d v\left(b^{\prime}\right) \\
& \geq \epsilon \int_{D_{T}} \frac{1}{\left|\mathcal{F}_{\rho\left(b^{\prime}\right)}\left(b^{\prime}\right)\right|} \operatorname{SUM}_{\rho\left(b^{\prime}\right)}^{\mathcal{F}}[v]\left(b^{\prime}\right) d v\left(b^{\prime}\right) .
\end{aligned}
$$

Since $E=D_{T}$ we now have

$$
\int_{D_{T}} u(b) d v(b) \geq \epsilon \int_{D_{T}} v(b) d v(b)
$$

which implies

$$
\begin{aligned}
& v_{v}\left(\left\{M_{T}^{\mathcal{F}}[u, v](b)>\epsilon\right\}\right)=v_{v}\left(D_{T}\right) \\
& \quad \leq \frac{1}{\epsilon} \int_{\left\{M_{T}^{\mathscr{F}}[u, v](b)>\epsilon\right\}} u(b) d v(b) \leq \frac{\|u\|_{L^{1}(B, v)}}{\epsilon} .
\end{aligned}
$$

The theorem follows by letting $T \rightarrow \infty$.

3.5. A ratio maximal inequality in $\boldsymbol{L}^{p}$. Let us note that the weak type $(1,1)$ maximal inequality can be used to derive a strong type $L^{p}$ maximal inequality, as follows.

Theorem 3.4. Let $u, v \in L^{1}(B, v)$ with $v>0$ on $B$, and let

$$
M^{\mathcal{F}}[u, v]:=\sup _{n}\left|\operatorname{RATIO}_{n}^{\mathcal{F}}[u, v]\right| .
$$

If $\mathcal{F}$ is anchored and satisfies the extreme Besicovich property then the strong type $L^{p}$ ratio maximal inequality holds (for $\left.1<p<\infty\right)$, namely, assuming $\frac{u}{v} \in L^{p}\left(v_{v}\right)$,

$$
\left\|M^{\mathcal{F}}[u, v]\right\|_{L^{p}\left(v_{v}\right)} \leq \frac{p}{p-1}\left\|\frac{u}{v}\right\|_{L^{p}\left(v_{v}\right)}
$$

or more explicitly

$$
\int_{B} M^{\mathcal{F}}[u, v]^{p}(b) v(b) d v \leq\left(\frac{p}{p-1}\right)^{p} \int_{B}\left(\frac{u(b)}{v(b)}\right)^{p} v(b) d v<\infty .
$$

Proof. Let us first recall the following basic fact (see [Ga70]). Suppose two nonnegative measurable functions $\Phi$ and $\Psi$ on a standard $\sigma$-finite measure space $(Y, \eta)$ satisfy 
(1) $\Psi \in L^{p}(Y, \eta)$, for some $1<p<\infty$,

(2) $\eta\{y ; \Phi(y)>\epsilon\}<\infty$ for all $\epsilon>0$,

(3) $\eta\{y ; \Phi(y)>\epsilon\} \leq \frac{1}{\epsilon} \int_{\{y ; \Phi(y)>\epsilon\}} \Psi(y) d \eta$.

Then $\Phi$ is in $L^{p}(Y, \eta)$, and $\|\Phi\|_{L^{p}(Y, \eta)} \leq \frac{p}{p-1}\|\Psi\|_{L^{p}(Y, \eta)}$.

Fixing $v>0$, let us consider the measure space $(Y, \eta)=\left(B, v_{v}\right)$, and define $\Phi=M^{\mathcal{F}}[u, v]$, and $\Psi=\frac{u}{v}$. Then if both $u$ and $v$ are in $L^{1}(v)$, we have by Theorem 3.3 that

$$
\begin{aligned}
\eta\{y ; \Phi(y)>\epsilon\} & =v_{v}\left\{M^{\mathcal{F}}[u, v](b)>\right\} \\
& \leq \frac{1}{\epsilon} \int_{\left\{M^{\mathscr{F}}[u, v](b)>\epsilon\right\}} \frac{u(b)}{v(b)} v(b) d v \\
& =\frac{1}{\epsilon} \int_{\{y ; \Phi(y)>\epsilon\}} \Psi(y) d \eta
\end{aligned}
$$

so that conditions 2 and 3 above are satisfied. Assuming in addition that $\frac{u}{v} \in L^{p}\left(v_{v}\right)$, condition 1 above is satisfied as well, and we can conclude that

$$
\left\|M^{\mathcal{F}}[u, v]\right\|_{L^{p}\left(v_{v}\right)} \leq \frac{p}{p-1}\left\|\frac{u}{v}\right\|_{L^{p}\left(v_{v}\right)}
$$

and the proof of the $L^{p}$ maximal inequality is complete.

Remark 3.5. Rather than assuming directly that the equivalence relation in question is hyperfinite, we have chosen to formulate the ratio theorems for equivalence relations satisfying extreme asymptotic invariance and the extreme Besicovich property. This was done in order to emphasize the tools we have used and their relation to classical arguments in amenable ergodic theory. It would be of course desirable to generalize the ratio ergodic theorem for equivalence relations with the sets $\mathscr{F}_{n}(b)$ satisfying the ordinary Besicovich property. On the other hand, the doubling condition introduced in [BN13a] or polynomial volume growth condition on the sets $\mathcal{F}_{n}(b)$ are insufficient for the validity of the ratio ergodic theorem. Indeed, the ratio ergodic theorem fails already for certain natural polynomially growing Følner sequences in actions of $\mathbb{Z}^{2}$. For more on this fact and for positive results on the ratio ergodic theorem and its connection to the Besicovich property of the underlying family of sets in the case of $\mathbb{Z}^{d}$-actions see [Ho10]. Further results on the interesting connection between the Besicovich property and the ratio ergodic theorem has very recently been obtained in the case where the equivalence relation is given by orbits of a countable group and the action is measure-preserving in [Ho13].

3.6. Cocycles and extensions of equivalence relations. Given an equivalence relation $\mathcal{R}$ and a sequence $\mathcal{F}$ of subset functions with extreme properties, one can use cocycles defined on $\mathscr{F}$ in order to define a sequence of subset functions with extreme properties defined on an extension of $\mathcal{R}$. This fact will be applied below to 
actions of free groups. In general, suppose $(X, \lambda)$ is a standard measure space, $\Gamma$ is a group acting by measure-class-preserving transformations on $X$ and $\alpha: \mathcal{R} \rightarrow \Gamma$ is a measurable cocycle. This means that $\alpha$ satisfies the cocycle equation

$$
\alpha\left(b_{1}, b_{2}\right) \alpha\left(b_{2}, b_{3}\right)=\alpha\left(b_{1}, b_{3}\right)
$$

for a.e. $b_{1}, b_{2}, b_{3} \in B$. Let $\mathcal{R}^{\alpha}=\mathcal{R}^{\alpha}(X)$ be the equivalence relation on $X \times B$ given by $(x, b) \sim_{\mathcal{R}^{\alpha}}\left(x^{\prime}, b^{\prime}\right)$ if and only $\left(b, b^{\prime}\right) \in \mathcal{R}$ and $\alpha\left(b^{\prime}, b\right) x=x^{\prime}$.

Lemma 3.6. Suppose $\mathscr{F}=\left\{\mathscr{F}_{n}\right\}_{n=1}^{\infty}$ is a sequence of subset functions for $\mathcal{R}$. Let $\mathscr{F}^{\alpha}=\left\{\mathscr{F}_{n}^{\alpha}\right\}_{n=1}^{\infty}$ be defined by

$$
\widetilde{F}_{n}^{\alpha}(x, b)=\left\{\left(x^{\prime}, b^{\prime}\right) \in X \times B: b^{\prime} \in \mathscr{F}_{n}(b), x^{\prime}=\alpha\left(b^{\prime}, b\right) x\right\} .
$$

If $\mathscr{F}$ is anchored then $\mathcal{F}^{\alpha}$ is anchored. If $\mathcal{F}$ is extremely asymptotically invariant then $\mathscr{F}^{\alpha}$ is extremely asymptotically invariant and if $\mathscr{F}^{\prime}$ has the extreme Besicovich property then $\mathscr{F}^{\alpha}$ has the extreme Besicovich property.

Proof. We will show that if $\mathscr{F}$ is extremely asymptotically invariant then $\mathscr{F}^{\alpha}$ is also extremely asymptotically invariant. The other claims are similar. Let $\Phi \subset \operatorname{Inn}(\mathcal{R})$ be a countable generating set witnessing the extreme asymptotic invariance of $\mathcal{F}$. For each $\phi \in \Phi$, define $\phi_{\alpha} \in \operatorname{Inn}\left(\mathcal{R}^{\alpha}\right)$ by $\left.\phi_{\alpha}(x, b)=(\alpha(\phi(b), b) x), \phi(b)\right)$. Then $\Phi_{\alpha}:=\left\{\phi_{\alpha}: \phi \in \Phi\right\} \subset \operatorname{Inn}\left(\mathcal{R}^{\alpha}\right)$ is a countable generating set witnessing the extreme asymptotic invariance of $\mathscr{F}^{\alpha}$.

\section{A horospherical ratio ergodic theorem for the free group}

As in the introduction, we let $\mathbb{F}=\left\langle a_{1}, \ldots, a_{r}\right\rangle$ denote the free group of rank $r \geq 2$, $\partial \mathbb{F}$ denote its boundary, $v$ the Markov measure on $\partial \mathbb{F}, \mathcal{R}_{0}$ the tail equivalence relation and $H_{\xi}$ the horosphere based at $\xi \in \partial \mathbb{F}$ passing through the identity.

Let $\mathscr{B}=\left\{\mathscr{B}_{n}\right\}$ be the sequence of subset functions given by

$$
\mathscr{B}_{n}(\xi)=\left\{\eta \in \partial \mathbb{F}: \eta_{k}=\xi_{k} \text { for all } k>n\right\} .
$$

In other words, $\mathscr{B}_{n}(\xi)$ is the set of all $g \xi$ where $g$ ranges over the intersection of the horosphere $H_{\xi}$ with the ball of radius $2 n$ in $\mathbb{F}$.

In the next subsection we show:

Theorem 4.1. The sequence $\mathcal{B}$ is anchored, extremely asymptotically invariant and satisfies the extreme Besicovich property. 


\subsection{Extreme properties of $\mathscr{B}$}

Definition 4.1. We will say that a map $\phi: \partial \mathbb{F} \rightarrow \partial \mathbb{F}$ has order $\leq n$ if there is a bijection $\phi^{\prime}: S^{n} \rightarrow S^{n}$ such that

(1) for any $\xi \in \partial \mathbb{F}$,

$$
\phi\left(\xi_{1}, \ldots, \xi_{n}\right)=\left(\xi_{1}^{\prime}, \ldots, \xi_{n}^{\prime}, \xi_{n+1}, \xi_{n+2}, \ldots\right)
$$

where $\phi^{\prime}\left(\xi_{1}, \ldots, \xi_{n}\right)=\left(\xi_{1}^{\prime}, \ldots, \xi_{n}^{\prime}\right)$;

(2) in the above $\xi_{n}^{\prime}=\xi_{n}$ and, since $\phi(\xi) \in \partial \mathbb{F}, \xi_{i+1}^{\prime} \neq\left(\xi_{i}^{\prime}\right)^{-1}$ for any $i$.

Lemma 4.2. The set of finite order inner automorphisms of $\mathcal{R}_{0}$ generates $\mathcal{R}_{0}$.

Proof. If $(\xi, \eta) \in \mathcal{R}_{0}$ then there exists an $N$ such that $n \geq N$ implies $\xi_{n}=\eta_{n}$. Let $\phi^{\prime}: S^{N} \rightarrow S^{N}$ be a bijection satisfying

(1) $\phi^{\prime}\left(\xi_{1}, \ldots, \xi_{N}\right)=\left(\eta_{1}, \ldots, \eta_{N}\right)$;

(2) if $\left(s_{1}, \ldots, s_{N}\right) \in S^{N}$ satisfies $s_{i+1} \neq s_{i}^{-1}$ for any $i$ and $\phi^{\prime}\left(s_{1}, \ldots, s_{N}\right)=$ $\left(t_{1}, \ldots, t_{N}\right)$ then $t_{i+1} \neq t_{i}^{-1}$ for any $i$.

Define $\phi \in \operatorname{Inn}\left(\mathcal{R}_{0}\right)$ by

$$
\phi\left(s_{1}, s_{2}, \ldots\right)=\left(t_{1}, \ldots, t_{N}, s_{N+1}, \ldots\right),
$$

where $\phi^{\prime}\left(s_{1}, \ldots, s_{N}\right)=\left(t_{1}, \ldots, t_{N}\right)$. This is an inner automorphism of finite order and clearly $\phi(\xi)=\eta$.

Proof of Theorem 4.1. It is immediate from the definition of $\mathscr{B}$ that $\mathcal{B}$ is anchored and satisfies the extreme Besicovich property. To see that it is extremely asymptotically invariant, let $\Phi \subset \operatorname{Inn}\left(\mathcal{R}_{0}\right)$ be the set of finite order inner automorphisms. This is a countable generating set by the previous lemma. Clearly, each $\mathscr{B}_{n}$ is preserved under all automorphisms of order $<n$.

4.2. A horospherical ratio pointwise ergodic theorem. Let $(X, \lambda)$ be a standard $\sigma$ finite measure space on which $\mathbb{F}$ acts non-singularly. Let $\alpha: \mathcal{R}_{0} \rightarrow \mathbb{F}$ be the cocycle $\alpha(\xi, \eta)=g \in \mathbb{F}$ where $g \eta=\xi$ (e.g., $g=\left(\xi_{1} \cdots \xi_{N}\right)\left(\eta_{1} \cdots \eta_{N}\right)^{-1}$ if $\xi_{n}=\eta_{n}$ for all $n \geq N)$. Define the equivalence relation $\mathcal{R}_{0}^{\alpha}=\mathcal{R}_{0}^{\alpha}(X)$ on $X \times \partial \mathbb{F}$ and the sequence of subset functions $\mathscr{B}^{\alpha}=\left\{\mathscr{B}_{n}^{\alpha}\right\}_{n=1}^{\infty}$ as in $\S$ 3.6. By Theorem 4.1 and Lemma 3.6, it follows that $\mathscr{B}^{\alpha}$ is anchored, extremely asymptotically invariant and satisfies the extreme Besicovich property. So Theorem 3.1 implies:

Theorem 4.2. $\mathscr{B}^{\alpha}$ is a ratio ergodic sequence in $L^{1}$. Namely, for every $u, v \in L^{1}(X \times$ $\partial \mathbb{F}, \lambda \times v)$ with $v>0$, $\operatorname{RATIO}_{n}^{\mathscr{P}^{\alpha}}[u, v]$ converges pointwise a.e. to $\mathbb{E}_{(v \times \lambda) v}\left[\frac{u}{v} \mid \mathcal{R}_{0}^{\alpha}\right]$. 
Theorem 2.1 is implied by Theorem 4.2 above because

$$
\mathscr{B}^{\alpha}(x, \xi)=\left\{g(x, \xi): g^{-1} \in H_{\xi},|g| \leq 2 n\right\}
$$

implies

$$
\operatorname{RATIO}_{n}^{\mathcal{B}^{\alpha}}[u, v]=\frac{\sum_{g^{-1} \in H_{\xi},|g| \leq 2 n} \hat{T}^{g} u(x, \xi)}{\sum_{g^{-1} \in H_{\xi},|g| \leq 2 n} \hat{T}^{g} v(x, \xi)} .
$$

Remark 4.3. The relation $\mathcal{R}_{0}$ defined in $\S 3$ is in fact the tail relation of a one sided Markov shift of finite type coding the boundary of the free group. The subset functions $\mathscr{B}_{n}(\xi)$ defined there are in fact finite equivalence classes associated with the $n$-step tail relations, namely equality of sequences from the $n$-th letter onwards. This observation implies that Theorem 2.1 and Theorem 4.2 admit far-reaching generalizations, to the case of the Markov shift of finite type associated with any Markov group. In particular, it is possible to prove analogs of these results for any non-elementary word-hyperbolic group with any set of generators. Ergodic theorems for Markov groups are developed in detail along these lines in [BN].

\section{Ergodic components of the horospherical relation: some examples}

Observe that the cocycle $\alpha: \mathcal{R}_{0} \rightarrow \mathbb{F}$ defined above takes values in $\mathbb{F}^{2}:=$ the index 2 subgroup of $\mathbb{F}$ consisting of all words of even reduced length. Let $\mathbb{F} \curvearrowright(X, \lambda)$ be a non-singular action on a standard $\sigma$-finite measure space. Theorem 4.2 raises the following two natural questions.

(1) If $\mathbb{F} \curvearrowright(X, \lambda)$ is ergodic then is the diagonal action $\mathbb{F} \curvearrowright(X \times \partial \mathbb{F}, \lambda \times v)$ ergodic?

(2) If the diagonal action $\mathbb{F}^{2} \curvearrowright(X \times \partial \mathbb{F}, \lambda \times \nu)$ is ergodic then is the equivalence relation $\mathcal{R}_{0}^{\alpha}(X)$ ergodic?

In [BN13a] it was shown that, if $(X, \lambda)$ is a probability space and the action preserves the measure then the answer to both questions is 'yes'. We show here that both questions have negative answers for actions on general non-singular actions on $\sigma$-finite measure spaces .

Example 5.1. This is a counterexample to (1). Let $X=(\partial \mathbb{F} \times \partial \mathbb{F}) \backslash \Delta$ be the set of pairs of distinct points in the boundary $\partial \mathbb{F}$, which can also be identified with set of biinfinite geodesics in the Cayley tree. As is well known, there exists a Radon measure $\lambda$ on $X$, which is absolutely continuous to the product measure $v \times v$ and invariant under the action of $\mathbb{F}$ on $X$ (given by the diagonal action on the product). Moreover $\mathbb{F} \curvearrowright(X, \lambda)$ is ergodic by Kaimanovich's double ergodicity Theorem [Ka03] (which can easily be proven directly in this special case). 
Consider now the space $X \times \partial \mathbb{F}$ with the product measure $v \times \lambda$, and the invariant conull set consisting of triples of distinct points. It is well-known that the action of $\mathbb{F}$ on the latter set is in fact proper (as an action on a metric space) and in particular admits a global Borel section. Since the equivalence relation $\mathcal{R}$ is a sub relation of the relation determined by the $\mathbb{F}$-orbits, it follows that $\mathcal{R}$ admits many $\mathcal{R}$-invariant sets of intermediate measure and is certainly not ergodic. In fact, the $\mathbb{F}$-action on the product $X \times \partial \mathbb{F}$ is measure-theoretically smooth.

Example 5.2. The following provides further counterexamples to (1). Let $G=$ $\mathrm{SL}_{2}(\mathbb{R})$, and $X=G / H$, with $H$ a unimodular connected subgroup, and $\lambda$ taken as the $G$-invariant measure on $G / H$. Let $\mathbb{F}$ act via a dense homomorphism $\pi: \mathbb{F} \rightarrow G$.

We want to consider the question of ergodicity of $\mathbb{F}$ on the product $X \times \partial \mathbb{F}$, and for that let us view the homomorphism $\pi$ as a cocycle $\alpha: \mathbb{F} \times \partial \mathbb{F} \rightarrow G$ via $\alpha(g, b)=$ $\pi(g)$. The Mackey range $Y$ of the cocycle is the space of ergodic components of the induced action of $\mathbb{F}$ on $\partial \mathbb{F} \times G$ (by $f(b, g)=(f b, \alpha(f, b) g)$ ). The group $G$ acts on $\partial \mathbb{F} \times G$ via $g(f, h)=\left(f, h g^{-1}\right)$. Because this action commutes with the action of $\mathbb{F}$, it descends to an action on $Y$. Since the action of $\mathbb{F}$ on $\partial \mathbb{F}$ is amenable and ergodic, $G$ acts amenably and ergodically on $Y$. By [Zi78b], any ergodic amenable action of $G$ factors over a transitive amenable action $G / L$, with $L$ being a closed amenable subgroup.

Since $L$ is amenable, it leaves a probability measure on the boundary of hyperbolic space invariant, and therefore it is either conjugate to a subgroup of the maximal compact subgroup $K$, or conjugate to a subgroup of the minimal parabolic subgroup $P$, or to a subgroup of the group $N_{K}(A)$, the normalizer in $K$ of the diagonal group $A$. By passing to a further factor if necessary, we can therefore assume either $L=K$, $L=P$ or $L=N_{K}(A)$.

Since $G / L$ is a factor of the Mackey range of the cocycle $\alpha$, it follows that $\alpha$ is cohomologous to a cocycle taking values in $L$, and this is equivalent to $\partial \mathbb{F}$ admitting an $\alpha$-invariant map $\psi$ to $G / L$. Since the cocycle is defined by $\alpha(g, b)=\pi(g)$, this amounts to a measurable non-singular map $\psi$, equivariant with respect to the actions of $\mathbb{F}$ on both sides.

Thus we have a non-singular factor map $\psi: \partial \mathbb{F} \rightarrow G / L$. Now in general (see [Zi78a]), for any $G$-space $X, \mathbb{F}$ is ergodic on $X \times \partial \mathbb{F}$ (acting via $\pi$ ) if and only if $G$ is ergodic on $Y \times X$. Choosing $X=G / H$, by Moore's ergodicity theorem (see e.g. [Zi84]), $G$ is ergodic on $G / L \times G / H$ if and only if $H$ is ergodic on $G / L$. Since the Mackey range $Y$ factors over $G / L$, it follows of course that $G$ is not ergodic on $Y \times G / H$ if $H$ is not ergodic on $G / L$. Choosing $H$ to be the trivial group $\{e\}$, the maximal compact subgroup $K$, or the diagonal subgroup $A$, we obtain that $H$ is not ergodic on $G / L$, for any of the admissible choices of $L$, namely $L=K, L=P$ or $L=N_{K}(A)$.

Thus taking $X$ to be the group itself $\left(X=\mathrm{SL}_{2}(\mathbb{R})\right)$, or the hyperbolic plane $\left(X=\mathbb{H}^{2}=G / K\right)$ or the de-Sitter space $(X=G / A)$, we conclude that the $\mathbb{F}$-action on $X \times \partial \mathbb{F}$ is not ergodic. 
Example 5.3. To obtain a counterexample to question (2), we let $X=\partial \mathbb{F}$ and consider the product action of $\mathbb{F}$ on $(\partial \mathbb{F} \times \partial \mathbb{F}, v \times v)$. This action is well-known to be ergodic as already noted in Example 5.1. The action restricted to the subgroup of even words $\mathbb{F}^{2}$ on $(\partial \mathbb{F} \times \partial \mathbb{F}, v \times v)$ is also ergodic. However, the equivalence relation $\mathcal{R}_{0}^{\alpha}(\partial \mathbb{F})$ is not ergodic. To see this, note that a point $(b, c) \in \partial \mathbb{F} \times \partial \mathbb{F}$ with $b \neq c$ determines a geodesic $\gamma: \mathbb{Z} \rightarrow \mathbb{F}$ from $b$ to $c$. Let $H_{c}$ be the horosphere centered at $c$ that passes through the identity element. Without loss of generality we may assume that $\gamma(0)$ is contained in $H_{c}$. Define $J_{(b, c)}(n)=\gamma(n)^{-1} \gamma(n+1)$. We claim that the map $(b, c) \in \partial \mathbb{F} \times \partial \mathbb{F} \mapsto J_{(b, c)}$ is invariant under the relation $\mathcal{R}_{0}^{\alpha}(\partial \mathbb{F})$. Because the map $(b, c) \mapsto J_{(b, c)}$ is not constant a.e. this proves that $\mathcal{R}_{0}^{\alpha}(\partial \mathbb{F})$ is not ergodic.

Any element in the $\mathcal{R}_{0}^{\alpha}(\partial \mathbb{F})$-equivalence class of $(b, c)$ has the form $\left(g^{-1} b, g^{-1} c\right)$ where $g \in H_{c}$. Notice that $g^{-1} H_{c}=H_{g^{-1}}$. Therefore, $g^{-1} \gamma$ is the geodesic from $g^{-1} b$ to $g^{-1} c$ and $g^{-1} \gamma(0)$ is contained in $H_{g^{-1}}$. So if $J^{\prime}=J_{\left(g^{-1} b, g^{-1} c\right)}$ then

$$
J^{\prime}(n)=\left(g^{-1} \gamma(n)\right)^{-1} g^{-1} \gamma(n+1)=\gamma(n)^{-1} \gamma(n+1)=J_{(b, c)}(n) .
$$

This proves the claim.

It would be useful to establish general conditions on an ergodic non-singular action $\mathbb{F} \curvearrowright(X, \lambda)$ which imply positive answers to questions (1) and (2) above. As am example, note that even the following is not known.

Question 5.4. Suppose $\mathbb{F} \rightarrow \operatorname{Isom}\left(\mathbb{E}^{n}\right)$ is a dense embedding into the isometry group of Euclidean $n$-space. Through this embedding, $\mathbb{F}$ acts by isometries on Euclidean $n$-space. Is the induced action $\mathbb{F} \curvearrowright\left(\mathbb{E}^{n} \times \partial \mathbb{F}, \lambda^{n} \times v\right)$ ergodic? Here $\lambda^{n}$ is the Lebesgue measure on $\mathbb{E}^{n}$. If so, then is the equivalence relation $\mathcal{R}_{0}^{\alpha}\left(\mathbb{E}^{n}\right)$ ergodic?

\section{References}

[AK63] V. I. Arnol'd and A. L. Krylov, Uniform distribution of points on a sphere and certain ergodic properties of solutions of linear ordinary differential equations in a complex domain. Dokl. Akad. Nauk SSSR 148 (1963), 9-12; English transl. Soviet Math. Dokl. 4 (1963), 1-5. Zbl 0237.34008 MR 0150374

[BK12] A. Bufetov and A. Klimenko, On Markov operators and ergodic theorems for group actions. Eur. J. Comb. 33 (2012), no. 7, 1427-1443. Zbl 1281.37004 MR 2923460

[BN13a] L. Bowen and A. Nevo, Geometric covering arguments and ergodic theorems for free groups. L'Enseignement Math. Enseign. (2) 59 (2013), no. 1-2, 133-164. Zbl 06238400 MR 3113602

[BN13b] L. Bowen. and A. Nevo, Pointwise ergodic theorems beyond amenable groups. Ergodic Theory Dynam. Systems. 33 (2013), no. 3, 777-820. Zbl 1276.37002 MR 3062901

[BN] L. Bowen and A. Nevo, Ergodic theorems for Markov groups. In preparation. 
[Br06] E. Breuillard, Random walks on Lie groups. Preprint 2006, available at www.math.u-psud.fr/ breuilla/part0gb.pdf

[Br10] E. Breuillard Equidistribution of dense subgroups on nilpotent Lie groups. Ergodic Theory Dynam. Systems 30 (2010), no. 1, 131-150. Zbl 1194.22012 MR 2586348

[Br05] E. Breuillard Local limit theorems and equidistribution of random walks on the Heisenberg group. Geom. Funct. Anal. 15 (2005), no. 1, 35-82. Zbl 1083.60008 MR 2140628

[Bu02] A. I. Bufetov, Convergence of spherical averages for actions of free groups. Ann. of Math. (2) 155 (2002), no. 3, 929-944. Zbl 1028.37001 MR 1923970

[CO60] R. V. Chacon and D. S. Ornstein, A general ergodic theorem. Illinois J. Math. 4 (1960), 153-160. Zbl 0134.12102 MR 0110954

[Fe07] J. Feldman, A ratio ergodic theorem for commuting, conservative, invertible transformations with quasi-invariant measure summed over symmetric hypercubes. Ergodic Theory Dynam. Systems 27 (2007), no. 4, 1135-1142. Zbl 1136.37005 MR 2342969

[Ga70] A. Garsia, Topics in almost everywhere convergence. Lectures in Adv. Math. 4, Markham Publishing Company, Chicago, Ill., 1970. Zbl 0198.38401 MR 0261253

[G03] A. Gorodnik, Lattice action on the boundary of $\operatorname{SL}(n, \mathbb{R})$. Ergodic Theory Dynam. Systems 23 (2003), no. 6, 1817-1837. Zbl 1050.22015 MR 2032490

[GM05] A. Gorodnik and F. Maucourant, Proximality and equidistribution on the Furstenberg boundary. Geom. Dedicata 113 (2005), 197-213. Zbl 1078.37007 MR 2171305

[GN12] A. Gorodnik and A. Nevo, On Arnold's and Kazhdan's equidistribution problems. Ergodic Theory Dynam. Systems 32 (2012), no. 6, 1972-1990 Zbl 1261.37006 MR 2995880

[GN14] A. Gorodnik and A. Nevo, Ergodic theory and the duality principle on homogeneous spaces. Geom. Funct. Anal. 24 (2014), no. 1, 159-244 MR 3177381

[GO07] A. Gorodnik and H. Oh, Orbits of discrete subgroups on a symmetric space and the Furstenberg boundary. Duke Math. J. 139 (2007), no. 3, 483-525. Zbl 1132.22012 MR 2350851

[GW07] A. Gorodnik and B. Weiss, Distribution of lattice orbits on homogeneous varieties. Geom. Funct. Anal. 17 (2007), no. 1, 58-115. Zbl 1112.37001 MR 2306653

[Gr99] R. I. Grigorchuk, Ergodic theorems for the actions of a free group and a free semigroup. Mat. Zametki 65 (1999), no. 5, 779-783; English transl. Math. Notes 65 (1999), no. 5-6, 654-657. Zbl 0957.22006 MR 1716245

[Gu69] Y. Guivarc'h, Généralisation d'un théorème de von-Neumann. C. R. Acad. Sci. Paris 268 (1969), 1020-1023. Zbl 0176.11703 MR 0251191

[Gu76] Y. Guivarc'h, Equirépartition dans les espaces homogènes. In Théorie ergodique (Actes Journées Ergodiques, Rennes, 1973/1974), Lecture Notes in Math. 532, Springer-Verlag, Berlin 1976, 131-142. Zbl 0368.28024 MR 0480860

[Ho10] M. Hochman, A ratio ergodic theorem for multiparameter non-singular actions. $J$. Eur. Math. Soc. (JEMS) 12 (2010), no. 2, 365-383. Zbl 1190.22007 MR 2608944

[Ho13] M. Hochman, On the ratio ergodic theorem for group actions. J. London Math. Soc. (2) 88 (2013), no. 2, 465-482. Zbl 06216293 MR MR3106731 
[Ho37] E. Hopf, Ergodentheorie. Ergeb. Math. Grengeb. 5, Springer-Verlag, Berlin 1937. Zbl 0017.28301

[Hu44] W. Hurewicz, Ergodic theorem without invariant measure. Ann. of Math. (2) 45 (1944), 192-206. Zbl 0063.02944 MR 0009427

[Ka03] V. A. Kaimanovich, Double ergodicity of the Poisson boundary and applications to bounded cohomology. Geom. Funct. Anal. 13 (2003), no. 4, 852-861. Zbl 1027.60038 MR 2006560

[Ka65] D. A. Kazhdan, Uniform distribution on a plane. Trudy Moskov. Mat. Obsc. 14 (1965), 299-305 (in Russian). Zbl 0225.26018 MR 0193187

[L99] F. Ledrappier, Distribution des orbites des réseaux sur le plan réel. C. R. Acad. Sci. Paris Sér. I Math. 329 (1999), no. 1, 61-64. Zbl 0928.22012 MR 1703338

[L01] F. Ledrappier, Ergodic properties of some linear actions. J. Math. Sci. 105 (2001), 1861-1875. Zbl 1004.37004 MR 1871151

[LP03] F. Ledrappier and M. Pollicott, Ergodic properties of linear actions of $(2 \times 2)$ matrices. Duke Math. J. 116 (2003), no. 2, 353-388. Zbl 1020.37009 MR 1953296

[LP05] F. Ledrappier and M. Pollicott, Distribution results for lattices in $\operatorname{SL}(2, \mathbb{Q} p)$. Bull. Braz. Math. Soc. (N.S.) 36 (2005), no. 2, 143-176. Zbl 1101.37005 MR 2152013

[Ne94] A. Nevo, Harmonic analysis and pointwise ergodic theorems for non-commuting transformations. J. Amer. Math. Soc. 7 (1994), 875-902. Zbl 0812.22005 MR 1266737

[NS94] A. Nevo and E. Stein, A generalization of Birkhoff's pointwise ergodic theorem. Acta Math. 173 (1994), no. 1, 135-154. Zbl 0837.22003 MR 1294672

[PS13] M. Pollicott and R. Sharp, Ergodic theorems for actions of hyperbolic groups. Proc. Amer. Math. Soc. 141 (2013), no. 5, 1749-1757. Zbl 1266.28008 MR 3020860

[Va12] P. Varjú, Random walks in Euclidean space. Preprint, arXiv:1205.3399.

[Vo04] Ya. B. Vorobets, On the uniform distribution of orbits of finitely generated groups and semigroups of plane isometries. Mat. Sb. 195 (2004), no. 2, 17-40; English transl. Sb. Math. 195 (2004), no. 1-2, 163-186. Zbl 1077.37024 MR 2068950

[Zi78a] R. Zimmer, Amenable ergodic group actions and an application to Poisson boundaries of random walks. J. Funct. Anal. 27 (1978), 350-372. Zbl 0391.28011 MR 0473096

[Zi78b] R. J. Zimmer, Induced and amenable ergodic actions of Lie groups. Ann. Sci. Ec. Norm. Sup. 11 (1978), 407-28. Zbl 0401.22009 MR 0521638

[Zi84] R. Zimmer, Ergodic theory and semisimple groups. Monogr. Math. 81, Birkhäuser, Basel 1984. Zbl 0571.58015 MR 0776417

Received August 3, 2012; revised January 31, 2013

L. Bowen, Mathematics Department, 1 University Station C1200, University of Texas, Austin, TX 78712, U.S.A.

E-mail: lpbowen@math.utexas.edu

A. Nevo, Mathematics Department, Technion - Israel Institute of Technology, Haifa, 32000, Israel

E-mail: anevo@tx.technion.ac.il 